Article

\title{
Different Magnitudes of Tensile Strain Induce Human Osteoblasts Differentiation Associated with the Activation of ERK1/2 Phosphorylation
}

Junfeng Zhu ${ }^{1}$, Xiaoling Zhang ${ }^{2}$, Chengtao Wang ${ }^{3}$, Xiaochun Peng ${ }^{1}$ and Xianlong Zhang ${ }^{1}{ }_{*}$

1 Department of Orthopaedics, Shanghai the 6th People's Hospital, Jiaotong University, Shanghai, 200233, China. E-Mails: zhujf2002@hotmail.com (J. Z.); xcpeng81@163.com (X. P.)

2 Institute of Health Science, Shanghai Institute for Biological Sciences, Chinese Academy of Sciences, Shanghai, 200025, China. E-Mail: xlzhang@sibs.ac.cn

3 School of Mechanical Engineering, Shanghai Jiaotong University, Shanghai, 200030, China. E-Mail: ctwang@sjtu.edu.cn

* Author to whom correspondence should be addressed; E-Mail: zx140@vip.sina.com; Tel. +86-21-64369181; Fax: +86-21-64701361

Received: 22 September 2008; in revised form: 19 November 2008 / Accepted: 25 November 2008 / Published: 26 November 2008

\begin{abstract}
Mechanical factors are related to periprosthetic osseointegration following total hip arthroplasty. However, osteoblast response to strain in implanted femurs is unclear because of the absence of accurate stress-measuring methods. In our study, finite element analysis was performed to calculate strain distribution in implanted femurs. 0.8$3.2 \%$ tensile strain was then applied to human osteoblasts. Higher magnitudes of strain enhanced the expression of osteocalcin, type I collagen, and Cbfa1/Runx2. Lower magnitudes significantly increased ALP activity. Among these, type I collagen expression increased with the activation of ERK1/2 phosphorylation in a strainmagnitude-dependent manner. Our study marks the first investigation of osteoblast response at different magnitudes of periprosthetic strain. The results indicate that the functional status of human osteoblasts is determined by strain magnitude. The strain distribution in the proximal region of implanted femur should be improved for osseointegration.
\end{abstract}

Keywords: Prosthesis; Strain; Finite element; Osteoblast; Differentiation. 


\section{Introduction}

Periprosthetic osseointegration following total hip arthroplasty (THA) plays a key role in the stability of cementless stems. Bone formation around prostheses has been investigated at tissue level both in animal experiments and clinical radiological follow-up. At a cellular level, osteoblasts, which are cells close to the bone-implant interface, play an important role in the postoperative osseointegration of stems by producing the mineralized matrix. Various mechanical stimulations such as stretch influenced the proliferation and differentiation of osteoblasts in vitro [1-2]. Although it has been reported that stress magnitude may contribute to the way cells respond to mechanical stimulation [3-4], the response to periprosthetic strain in implanted femurs is unclear because of the absence of accurate stress-measuring methods. Recently, finite element analysis (FEA) has been a powerful tool used frequently to calculate the tissue strains in biomechanical studies [5]. Unfortunately, among these, there is limited research on the mechanical response of osteoblasts. In our study, a CT-based finite element model of the implanted human femur was built for analyzing the tissue-level strain magnitude at the bone-implant interface. The results of FEA were amplified 20 times to stimulate human osteoblasts in vitro. Our study marks the first investigation of osteoblast response at different magnitudes of periprosthetic strain. Given that osteoblast is the only cell in body that is capable of producing new bone, the information on strain sensitivity of osteoblasts may provide clues for speculating the status of periprosthetic osseointegration. Decreased periprosthetic osseointegration as well as osteoclast-mediated resorption was recognized as an important factor influencing implant survival time. Therefore, the information from this study may contribute to the development of a strategy for reducing the risk of prosthesis loosening.

\section{Results and Discussion}

Tissue-level strains in intact human bone are usually less than $1000 \mu \varepsilon$ [6]. After the insertion of an untapered femoral prosthesis, a peak strain of $1868 \mu \varepsilon$ was reported near the tip [7]. Figure 1A shows 29,841 elements in the THA finite element model, including 4,193 elements at the bone-implant interface. Owing to the improvement of prosthetic shape, a lesser peak strain of $1583 \mu \varepsilon$ was found in our study. From $375 \mu \varepsilon$ to $1583 \mu \varepsilon$, the interface bone strain increased along the stem axis. (Figure 1B) Similar strain pattern was stated in measuring the surface strains of the implanted femur [8]. We focused on the response of human osteoblast to different magnitudes of strain the bone-implant interface, which was important for the postoperative stability of cementless stems. However, most investigations have demonstrated that the magnitude of tissue-level strain was too low to induce cell response. The reason is that the strain loaded on cells inside the bone is different from the tissue-level strain calculated by FEA. There has been a strain amplification mechanism on osteoblasts inside the bone which mainly caused by the fluid flow around the osteoblasts [9]. Recently, a quantitative strain model at cellular level stated that strains loaded on the whole bone might be amplified over 20 times at the membrane of osteoblasts [10]. The amplification factor was calculated as a function of the load frequency and extensively justified to induce effective mechanical response of cells. For example, 0.3$5 \%$ tensile strain was produced according to the quantitative model and significantly induced the expression of osteoblast-specific genes in osteoblasts [10-12]. Based on the information, the strain 
magnitude at tissue level can be converted to the corresponding magnitude at cellular level. Here, the tissue-level strain of $375 \mu \varepsilon \sim 1583 \mu \varepsilon$ was amplified 20 times to stimulate human osteoblasts in vitro.

Figure 1. The tissue-level strain magnitude at the bone-implant interface (A) A threedimensional finite element (FE) model of the implanted human femur. 29,841 elements were generated in the FE model, including 4,193 elements at the bone-stem interface. (B) The strain distribution at the bone-implant interface. From $375 \mu \varepsilon$ to $1,583 \mu \varepsilon$, the interface bone strain increased along the stem axis. A peak strain of $1,583 \mu \varepsilon$ was found near the prosthetic tip. L: the level of the prosthetic tip. The white space below the prosthetic tip is the medullary cavity.

A

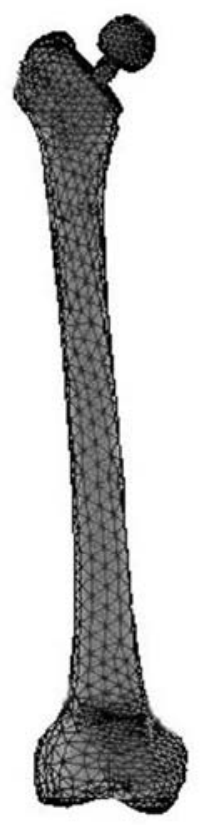

$\mathrm{B}$

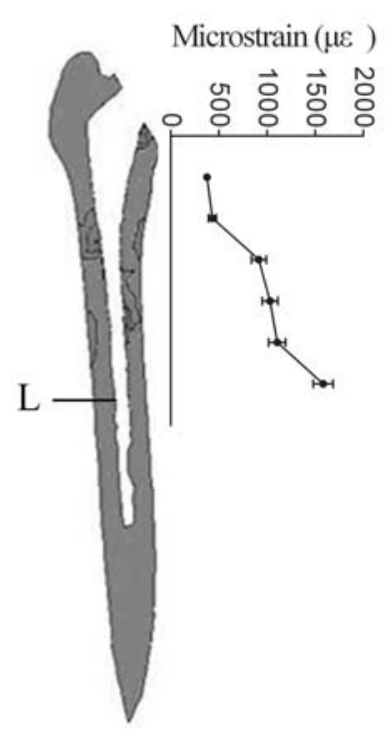

ALP activity, expression of type I collagen and Cbfa1/Runx2 were suggested to be associated with the magnitude of strain $[12,13]$. ALP activity and osteocalcin are considered as the indicators of osteogenesis. In bone marrow stromal cells, ALP activity increased at 5\% elongation, then decreased at $10 \%$ and $15 \%$ elongation [13]. Figure 2 showed that the ALP activity of human osteoblasts increased at $0.8 \%$ and $1.6 \%$ elongation, but remained unchanged at higher magnitudes of strain. In contrast, higher magnitudes of strain $(2.4 \%$ and $3.2 \%$ ) played a positive role in the expression of osteocalcin, while $0.8 \%$ and $1.6 \%$ elongation had no effects (Figure 3). Synthesized and secreted exclusively by osteoblasts at the late stages of maturation, osteocalcin regulates bone growth by binding to hydroxyapatite crystals, the key mineral component of bone [14]. At the mineralization stage, ALP activity decreased, but the expression of hydroxyapatite deposition-related genes such as osteocalcin increased in osteoblasts. Therefore, higher magnitudes of strain could further promote the functional status of osteoblasts by enhancing extracellular matrix maturation. This finding suggests that the osteoblasts around prosthetic tip may show more active functional status than those around the proximal part of prosthesis, since the interface bone strain increased along the stem axis in FEA model. Based on the foregoing results of FEA, the osteoblasts around the proximal part of prosthesis might be 
inhibited at the early stage of differentiation. This difference may lead to decreased bone formation around the proximal part of prosthesis. The dual characters of osteoblasts in response to mechanical strain were different from their progenitor cells. Those cells mostly showed an increase of ALP activity and responded to a broader magnitude spectrum. For example, $0.8 \%$ and $5 \%$ elongation both significantly increased the ALP activity of bone marrow stromal cells, but this had no effects on the expression of osteocalcin [13]. The differential regulation of ALP activity and osteocalcin expression might be attributed to the differential activation of various signaling pathways including cytoskeletal integrin rearrangement, stretch-activation of cation channels, and mitogen-activated protein kinases (MAPK) family.

Figure 2. Lower magnitudes of tensile strain increased ALP activity. ALP activity increased at $0.8 \%$ and $1.6 \%$ elongation, but remained unchanged at higher magnitudes of strain. ALP activity per sample was normalized for the total protein content of the cell lysate. Results are presented as the percentage of activity change as compared to the control group. “*” indicates $\mathrm{P}<0.01$.

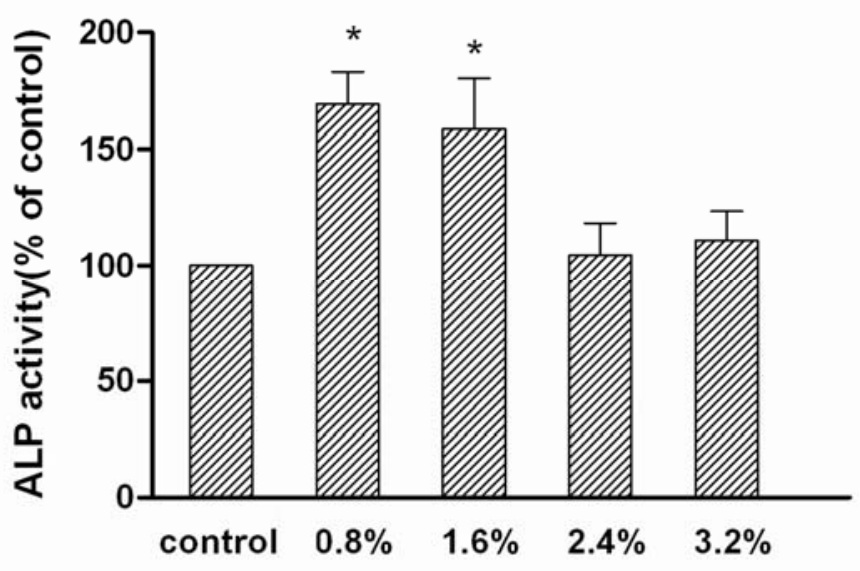

Type I collagen accounts for $90 \%$ of bone matrix proteins [15]. Most investigations have demonstrated that mechanical strain stimulated the expression of type I collagen [12, 13]. Differential responses were observed in our study. As compared to static control, the mRNA level of type I collagen increased in a strain-magnitude-dependent manner (Figure 3). The increasing strain gradually enhanced the expression of type I collagen in human osteoblast. As the initial step in bone tissue formation, the synthesis of type I collagen provides organic scaffold for the subsequent deposition of mineral. New bone mass can only be acquired by increased matrix synthesis [16]. In term of this viewpoint, higher magnitudes of strain would enhance periprosthetic bone formation by initiating the increase of type I collagen synthesis in human osteoblasts. Associated with the FEA result of the THA model, the expression of type I collagen was consistent with a postoperative histological phenomenon that hyperostosis was observed near the tip of the untapered stem where postoperative strain distribution markedly increased. Meanwhile, bone loss took place around the proximal part of femoral prosthesis where strain magnitude robustly decreased. All these evidences indicate that lower magnitudes of strain which are mainly located around the proximal part of prosthesis will lead to decreased bone formation 
compared with that of higher magnitudes; this may be an important factor influencing implant survival time. Therefore, the adverse strain distribution in the proximal region of implanted femurs should be targeted for reducing the risk of prosthesis loosening. Many FEA models showed that the strain distribution in implanted femurs is related to the stiffness and shape of the prosthesis.

Figure 3. Higher magnitudes of tensile strain induced the expression of osteocalcin (OC), type I collagen and Cbfa1/Runx2 in human osteoblasts. A. Agarose gel electrophoresis of PCR products. B. Semiquantitative results of osteocalcin, type I collagen, and Cbfa1/Runx2 gene normalized for GAPDH expression. $\mathrm{P}<0.01$, * as compared to static control, ++ as compared to the group with $1.6 \%$ elongation.
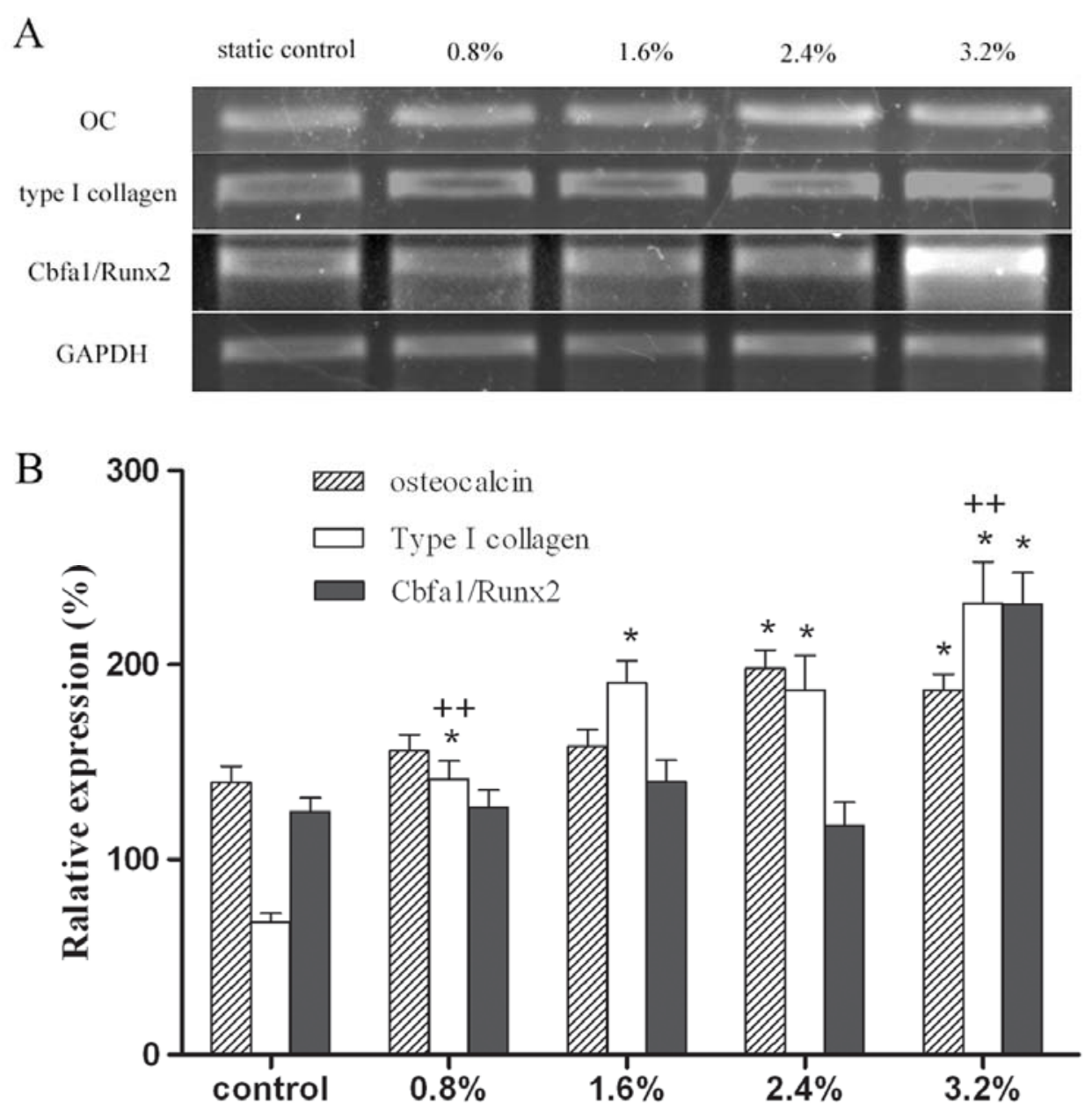

The use of a lower modulus or composite femoral stem might allow better load sharing and ultimately achieve a more stable biomechanical harmony [17]. Owing to excessive interfacial micromotion, the clinical results of this kind of system such as a Proplast-coated prosthesis were unsatisfactory in previous investigations. However, in term of the osteoblast response in this study, the lower-modulus femoral component might still have potential advantage if its interfacial micromotion could be improved. Cbfa1/Runx2 is a key transcription factor which regulates osteoblasts differentiation. As the end point of the MAPK signaling pathway, Cbfa1/Runx2 regulated the 
expression of osteoblast-specific genes after binding to osteoblast-specific cis-acting element [18]. Mechanical strain could increase [13, 18] or decrease [19] the Cbfa1/Runx 2 mRNA level. Figure 3 shows that the Cbfa1/Runx2 mRNA increased only at the highest magnitude of strain in human osteoblasts. At 3.2\% elongation, mechanical strain plays a positive role in the expression of osteocalcin and type I collagen. However, the expression of these genes and ALP activity also increased at other lower magnitudes, while the expression of Cbfa1/Runx 2 remained unchanged (Figures 2, 3). Similarly, the absence of Cbfa1/Runx2 expression was observed at protein level (data not shown). This phenomenon was not consistent with the foregoing "end point" theory on MAPK signaling pathway. The results suggest the presence of mechano-transduction threshold in the stretchinduced expression of Cbfa1/Runx 2 and indicate that Cbfa1/Runx 2 may not be indispensable for the expression of osteoblast-specific genes. A crosstalk pathway and other principal regulators might be involved in the mechanical signal transduction.

Figure 4. The effect of different magnitudes of strain on MAPK activity. Western blot of MAPK was shown as A. p-ERK and ERK, B. p-JNK and JNK, and C. p-p38 and p38. ERK1/2 was phosphorylated in a load-dose-dependant manner, whereas JNK and P38 were unaffected. D. Quantification graph for the protein levels of the phosphorylated kinases. The results were normalized to total kinase levels and expressed as a ratio to corresponding static control. $\mathrm{P}<0.01$, * as compared to the static control, ++ as compared to the group with $1.6 \%$ elongation.

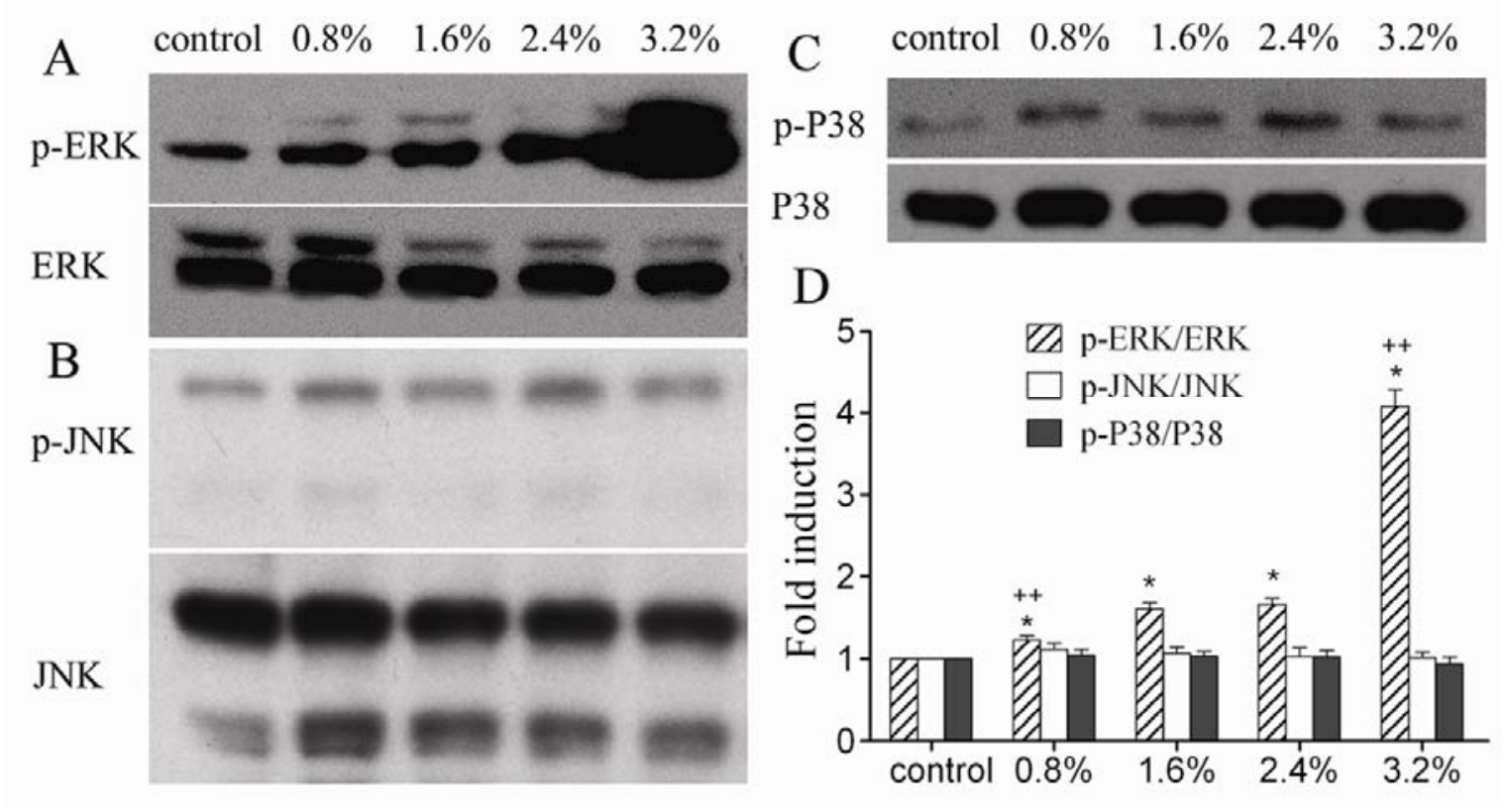

As MAPK family members, extracellular signal-regulated kinases (ERK1/2), c-jun N-terminal kinase (JNK), and p38MAPK were activated upon mechanical stimulation of the cells in several studies [16, 20, 21]. However, the reported phosphorylation of these members widely differed. Figure 4 shows that ERK1/2 was phosphorylated in a load-dose-dependant manner, whereas JNK and P38 were unaffected. This result was different from previous report that only JNK was activated by $9 \%$ tensile force applied in human osteoblasts [20]. All these evidences indicate that the selective 
activation of MAPK family members is related to the strain magnitude. This finding might justify the differential responses of human osteoblasts at different magnitudes of periprosthetic strain. It appears that the strain magnitudes used in our study were excluded from the magnitude spectrum for activation of JNK and P38 phosphorylation. The results showed that the increased expression of osteoblastspecific genes in human osteoblasts was associated with the activation of ERK1/2 phosphorylation. Interestingly, the activation of ERK1/2 phosphorylation in our study was highly consistent with the stretch-induced expression of type I collagen.(Figure 3B, 4D) Whether ERK1/2 signaling pathway plays a leading role in the mechanical induction of collagen gene requires further investigation. If it is true, the ERK1/2 signaling pathway might be a potential target for improving the overall integration of prosthesis.

\section{Experimental Section}

\subsection{Finite element (FE) model}

FE models of European femora have been extensively investigated. However, significant dimensional differences existed between the proximal femora of Asians and Caucasians [22, 23]. Smaller offset, narrower canal especially at the isthmus, and differential endosteal shape were found in Chinese $[24,25]$. In term of the ethnicity and skeletal characteristics of our target patients, a Chinese human femur was selected from 120 embalmed specimens, which was considered average in shape and bone density. The placement of a cementless titanium-alloy stem was performed according to the surgical manual. The CT scan images of the model were transferred to a graphics computer program. Based on the contours of the cortical bone, cancellous bone, and prosthesis, a three-dimensional FE model was constructed with a 10-node tetrahedral element. CONTA174 and TARGE170 elements were used to model the contact interface. The titanium stem and bone were assumed to be isotropic and linear elastic. The Poisson's ratios for the stem and bone were set to be 0.3. Young's modulus of $107 \mathrm{GPa}$ and $17 \mathrm{GPa}$ [10] were assigned to the stem and cortical bone. Young's modulus of the cancellous bone was linearly converted from CT Hounsfield values [26, 27]. The joint load of $1631 \mathrm{~N}$ and muscle forces were applied based on published data $[28,29]$. The strain distribution at the boneimplant interface was analyzed using FE software ANSYS 5.7.

\subsection{Cell culture}

SV40 human osteoblasts (sv40hOB, ATCC) were obtained from the Shanghai Institutes for Biological Sciences, Chinese Academy of Science, P.R. China. The mechanical sensitivity of sv40hOB cells was verified in previous studies [2, 30]. Cells were seeded to six-well BioFlex culture plates (Flexcell International, Hillsborough, NC, USA) with flexible membranes coated with collagen $\mathrm{I}$, at a density of $3.0 \times 10^{4}$ cells $/ \mathrm{cm}^{2}$. They were cultured in DMEM/F12 medium $(2 \mathrm{~mL})$ supplemented with G418 (0.3 mg/mL), ascorbic acid (50 mg/L), $10 \mathrm{mM} \beta$-glycerophosphate (Sigma, St. Louis, MO, USA) and $10 \%$ fetal bovine serum (HyClone, USA). Finally, they were incubated under $5 \% \mathrm{CO}_{2}$ atmosphere at $37{ }^{\circ} \mathrm{C}$ for four days to reach $90 \%$ confluence. The medium was changed every two days. 


\subsection{Application of mechanical strain}

Radial and circumferential strain was provided by an FX-4000T Flexcell BioFlex Tension Plus Unit (Flexcell International, USA). BioFlex culture plates were placed on a $25 \mathrm{~mm}$ diameter loading station. When vacuum pressure was applied to the plates through a vacuum pump, the membrane was deformed to create regulated strain. After reaching 90\% confluence, the cells were respectively subjected to tensile strains of $0.8 \%(7,500 \mu \varepsilon), 1.6 \%(16,000 \mu \varepsilon), 2.4 \%(24,000 \mu \varepsilon)$, and 3.2\% $(31,660$ $\mu \varepsilon)$ for $48 \mathrm{~h}$. Static controls were not stretched. One-half sine wave and a work frequency of $1 \mathrm{~Hz}$ were selected in this experiment.

\subsection{Alkaline phosphatase (ALP) activity assay}

ALP activity was assessed as described by Kasten [31]. The supernatant was incubated at $37{ }^{\circ} \mathrm{C}$ for $15 \mathrm{~min}$ with p-nitrophenyl phosphate. The coloring reaction was measured at $405 \mathrm{~nm}$. Protein concentration was determined with a bicinchoninic acid (BCA) protein assay kit (Pierce Chemical, USA). ALP activity per sample was normalized to the amount of total protein and displayed relative to the control.

\subsection{Semi-quantitative RT-PCR}

After being stretched, total RNA was extracted from the cells by using TRIzol reagent (Invitrogen, USA) according to the manufacturer's instructions. Then $1 \mu \mathrm{g}$ RNA was reverse-transcribed for first strand cDNA synthesis (RevertAidTM M-MuLV, Fermentas, USA). PCR amplification was performed using the primers listed in Table 1. RT-PCR products were electrophoresed on 1\% agarose gel with $0.5 \mathrm{mg} / \mathrm{mL}$ ethidium bromide. Bands were detected by UV illumination of ethidium bromidestained gels. Band intensities were quantitatively analyzed by Quantity One software for each gene and were normalized to the corresponding GAPDH values.

Table 1. Primer sequences and cycle conditions used for RT-PCR.

\begin{tabular}{llcc}
\hline \multicolumn{1}{c}{ Gene } & Primer sequence(Forward/Reverse) & T annealing & Cycles \\
\hline GAPDH & 5' GTTCCAATATGATTCCACCC 3' $^{\prime}$ & $52^{\circ} \mathrm{C}$ & 21 \\
& 5' AGGGATGATGTTCTGGAGAG 3' & & \\
Type I & 5' ACAGCCGCTTCACCTACAGC 3' & \multirow{2}{*}{$52^{\circ} \mathrm{C}$} & 22 \\
collagen & 5' TGCACTTTTGGTTTTTGGTCAT 3' & & \\
Osteocalcin & 5' GCCTTTGTGTCCAAGC 3' & $51^{\circ} \mathrm{C}$ & 30 \\
& 5' GGACCCCACATCCATAG 3' & & \\
Cbfa1/Runx2 & 5' TACCTGAGCCAGATGACG 3' & $58^{\circ} \mathrm{C}$ & 28 \\
\hline
\end{tabular}




\subsection{Western blot analysis of MAPK}

After being stretched, the cells were rinsed with PBS and lysed in SDS sample buffer $(0.2 \mathrm{~mL} ; 62.5$ $\mathrm{mM}$ Tris- $\mathrm{HCl}, \mathrm{pH} 6.8,2 \% \mathrm{SDS}, 10 \%$ glycerol, $50 \mathrm{mM}$ DTT, and $0.1 \%$ bromphenol blue). The samples were kept on ice and then boiled for $5 \mathrm{~min}$. Cells lysates were separated by $10 \%$ SDS-polyacrylamide gel electrophoresis and electrotransferred to polyvinylidene difluoride membranes (Millipore, USA). After being blocked with 5\% skim milk for $2 \mathrm{~h}$ at room temperature, the membrane was probed overnight at $4{ }^{\circ} \mathrm{C}$ with anti-ERK, anti-JNK, anti-p38, anti-phospho-p38, anti-phospho- ERK, and antiphospho-JNK(Cell Signaling, USA), respectively. After extensive washing, the membranes were incubated for $1 \mathrm{~h}$ at room temperature with an anti-rabbit secondary antibody conjugated to horseradish peroxidase. Immunoreactive bands were detected by an enhanced chemiluminescence system (Pierce) followed by exposure to X-OMAT Kodak films. The density of the bands was analyzed, and the results were normalized to total ERK1/2, JNK, and p38 MAPK.

\subsection{Statistical analysis}

All assays were repeated in two independent experiments with a minimum of $n=3$ for each data point. Statistical analysis among groups was performed by ANOVA and SNK test using SAS6.12 software package (SAS Ltd., NC, USA). Statistically significant values were defined as $P<0.05$.

\section{Conclusions}

The stretch-induced expression of osteoblast-specific genes in human osteoblasts is dependent on strain magnitude and associated with the activation of ERK1/2 phosphorylation. Higher magnitudes of periprosthetic strain increased the mRNA levels of osteocalcin, Cbfa1/Runx2, and type I collagen; this might owe to the differential level of ERK1/2 phosphorylation. Based on the results of FEA, lower magnitudes of strain which are mainly located around the proximal part of prosthesis compromise the functional status of human osteoblasts and contribute to decreased bone formation around the proximal part of prosthesis. The decreased periprosthetic osseointegration will make space for wear particles and lead to aseptic prosthesis loosening following THA. Based on this finding, the use of a lower modulus or composite femoral stem might still have potential advantage if its interfacial micromotion could be improved. In addition, a potential crosstalk pathway and some principal regulators need to be further investigated. If the vital role of ERK1/2 phosphorylation in the mechanical response of human osteoblasts can be further supported by more preclinical trials, a special biological target may be proposed for reducing the risk of prosthesis loosening following THA.

\section{Acknowledgements}

We thank Dr. Zhihu Qu and Dr. Jieli Li for their technical assistance. The research was financially supported by the National Natural Science Foundation of China (No. 30470455). 


\section{References}

1. Danciu, T.E.; Adam, R.M.; Naruse, K.; Freeman, M.R.; Hauschka, P.V. Calcium regulates the PI3K-Akt pathway in stretched osteoblasts. FEBS Lett. 2003, 536, 193-197.

2. Weyts, F.A.; Bosmans, B.; Niesing, R.; Leeuwen, J.P.; Weinans, H. Mechanical control of human osteoblast apoptosis and proliferation in relation to differentiation. Calcif. Tissue Int. 2003, 72, 505-512.

3. Yamamoto, N.; Fukuda, K.; Matsushita, T.; Matsukawa, M.; Hara, F.; Hamanishi, C. Cyclic tensile stretch stimulates the release of reactive oxygen species from osteoblast-like cells. Calcif. Tissue Int. 2005, 76, 433-438.

4. Fan, X.; Rahnert, J.A.; Murphy, T.C.; Nanes, M.S.; Greenfield, E.M.; Rubin, J. Response to mechanical strain in an immortalized pre-osteoblast cell is dependent on ERK1/2. J. Cell Physiol. 2006, 207, 454-460.

5. Speirs, A.; Heller, M.; Duda, G.; Taylor, W. Physiologically based boundary conditions in finite element modeling. J. Biomech. 2007, 40, 2318-2323.

6. Fritton, S.P.; Kenneth, J.M.; Rubin, C.T. Quantifying the strain history of bone: spatial uniformity and self-similarity of low magnitude strains. J. Biomech. 2000, 33, 317-325.

7. Estok, D.M.; Harris, W.H. A stem design change to reduce peak cement strains at the tip of cemented total hip arthroplasty. J. Arthroplasty 2000, 15, 584-589.

8. Tai, C.L.; Lee, M.S.; Chen, W.P.; Hsieh, P.H.; Lee, P.C.; Shih, C.H. Biomechanical comparison of newly designed stemless prosthesis and conventional hip prosthesis -An experimental study. Biomed. Mater. Eng. 2005, 15, 239-249.

9. Cowin, S.C.; Weinbaum, S. Strain amplification in the bone mechanosensory system. Am. J. Med. Sci. 1998, 316, 184-188.

10. You, L.; Cowin, S.C.; Mitchell, B.S.; Mitchell, B.S.; Weinbaum, S.A. Model for strain amplification in the actin cytoskeleton of osteocytes due to fluid drag on pericellular matrix. $J$. Biomech. 2001, 34, 1375-1386.

11. Fong, K.D.; Nacamuli, R.P.; Loboa, E.G.; Henderson, J.H.; Fang, T.D.; Song, H.M.; Cowan, C.M.; Warren, S.M.; Carter, D.R. Longaker, M.T. Equibiaxial tensile strain affects calvarial osteoblast biology. J. Craniofac. Surg. 2003, 14, 348-355.

12. Liu, X.H.; Zhang, X.L.; Luo, Z.P. Strain-related collagen gene expression in human osteoblastlike cells. Cell Tissue Res. 2005, 322, 331-334.

13. Koike, M.; Shimokawa, H.; Kanno, Z.; Ohya, K.; Soma, K. Effects of mechanical strain on proliferation and differentiation of bone marrow stromal cell line ST2. J. Bone Miner. Metab. 2005, 23, 219-225.

14. Sila-Asna, M.; Bunyaratvej, A.; Maeda, S.; Kitaguchi, H.; Bunyaratavej, N. Osteoblast differentiation and bone formation gene expression in strontium-inducing bone marrow mesenchymal stem cell. Kobe J. Med. Sci. 2007, 53, 25-35.

15. Hakala, M.; Aho, K.; Aman, S.; Luukkainen, R.; Kauppi, M.; Risteli, J. Type I collagen degradation does not diminish with RA disease duration. Ann. Rheum. Dis. 2001, 60, 420-422.

16. Hatton, J.P.; Pooran, M.; Li, C.F.; Luzzio, C.; Hughes-Fulford, M. A short pulse of mechanical force induces gene expression and growth in MC3T3-E1 osteoblasts via an ERK 1/2 pathway. $J$. Bone Miner. Res. 2003, 18, 58-66. 
17. Maistrelli, G.L.; Fornasier, V.; Binnington, A.; McKenzie, K.; Sessa, V.; Harrington, I. Effect of stem modulus in a total hip arthroplasty model. J Bone Joint Surg. Br. 1991, 73, 43-46.

18. Ziros, P.G.; Gil, A.P.; Georgakopoulos, T.; Habeos, I.; Kletsas, D.; Basdra, E.K.; Papavassiliou, A.G. The bone specific transcriptional regulator Cbfal is a target of mechanical signals in osteoblastic cells. J. Biol. Chem. 2000, 277, 23934-23941.

19. Nikolovski, J.; Kim, B.S.; Mooney, D.J. Cyclic strain inhibits switching of smooth muscle cells to an osteoblast-like phenotype. FASEB J. 2003, 17, 455-457.

20. Matsuda, N.; Morita, N.; Matsuda, K; Watanabe, M. Proliferation and differentiation of human osteoblastic cells associated with differential activation of MAP kinases in response to epidermal growth factor, hypoxia, and mechanical stress in vitro. Biochem. Biophys. Res. Commun. 1998, 249, 350-354.

21. Lewthwaite, J.C.; Bastow, E.R.; Lamb, K.J.; Blenis, J.; Wheeler-Jones, C.P.D.; Pitsillides, A.A. A specific mechanomodulatory role for p38 MAPK in embryonic joint articular surface cell MEKERK pathway regulation. J. Biol. Chem. 2006, 281, 11011-11018.

22. Khang, G.; Choi, K.; Kim, C.S.; Yang, J.S.; Bae, T.S. A study of Korean femoral geometry. Clin. Orthop. Relat. Res. 2003, 406, 116-122.

23. Marshall, L.M.; Zmuda, J.M.; Chan, B.K.; Barrett-Connor, E.; Cauley, J.A.; Ensrud, K.E.; Lang, T.F.; Orwoll, E.S. Race and ethnic variation in proximal femur structure and BMD among older men. J. Bone Miner. Res. 2008, 23,121-130.

24. Fang, D.; Chiu, K.Y.; Kemedios, I.D.; Yin, Q. Osteometry of the Chinese proximal femur. J. Orthop. Surg. 1996, 4, 41-45.

25. Chiu, K.Y.; Fang, D. Endosteal shape of the proximal femur in Chinese. J. Orthop. Surg. 1997, 5, 21-24.

26. Couteau, B. Finite element modelling of the vibrational behaviour of the human femur using CTbased individualized geometrical and material properties. J. Biomech. 1998, 31, 383-386.

27. Radcliffe, I.A.J.; Prescott, P.; Man, H.S.; Taylor, M. Determination of suitable sample sizes for multi-patient based finite element studies. Med. Eng. Phys. 2007, 29, 1065-1072.

28. Bergmann, G.; Deuretzbacher, G.; Heller, M.; Graichen, F.; Rohlmann, A.; Strauss, J.; Duda, G.N. Hip contact forces and gait patterns from routine activities. J. Biomech. 2001, 34, 859-871.

29. Wong, A.S.; New, A.M.R. Isaacs, G.; Taylor, M. Effect of bone material properties on the initial stability of a cementless hip stem: a finite element study. Proc. Inst. Mech. Eng. 2005, 219, 265-275.

30. Jansen, J.H.; Weyts, F.A.; Westbroek, I.; Jahr, H.; Chiba, H.; Pols, H.A.P.; Verhaar, J.A.N.; van Leeuwen, J.P.T.M.; Weinans, H. Stretch-induced phosphorylation of ERK1/2 depends on differentiation stage of osteoblasts. J. Cell Biochem. 2004, 93, 542-551.

31. Kasten, P.; Luginbuhl, R.; van Griensven, M.; Barkhausen, T.; Krettek, C.; Bohner, M.; Bosch, U. Comparison of human bone marrow stromal cells seeded on calcium-deficient hydroxyapatite, beta-tricalcium phosphate and demineralized bone matrix. Biomaterials 2003, 24, 2593-2603.

(C) 2008 by the authors; licensee Molecular Diversity Preservation International, Basel, Switzerland. This article is an open-access article distributed under the terms and conditions of the Creative Commons Attribution license (http://creativecommons.org/licenses/by/3.0/). 